Supporting Information for:

\title{
Strong Spin-Selective Optical Stark Effect in Lead Halide Perovskite Quantum Dots
}

Yulu Li, ${ }^{1,2}$ Shan $\mathrm{He}^{1}$, Xiao Luo ${ }^{1}$ Xin $\mathrm{Lu}^{2}$ and Kaifeng $\mathrm{Wu}^{1 *}$

${ }^{1}$ State Key Laboratory of Molecular Reaction Dynamics and Dynamics Research Center for Energy and Environmental Materials, Dalian Institute of Chemical Physics, Chinese Academy of Sciences, Dalian, Liaoning 116023, China

${ }^{2}$ Department of Chemistry, College of Chemistry and Chemical Engineering, Xiamen University, Xiamen, Fujian 361005, China 
a

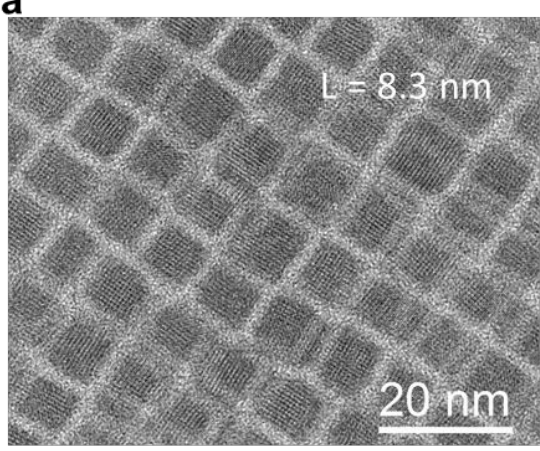

C

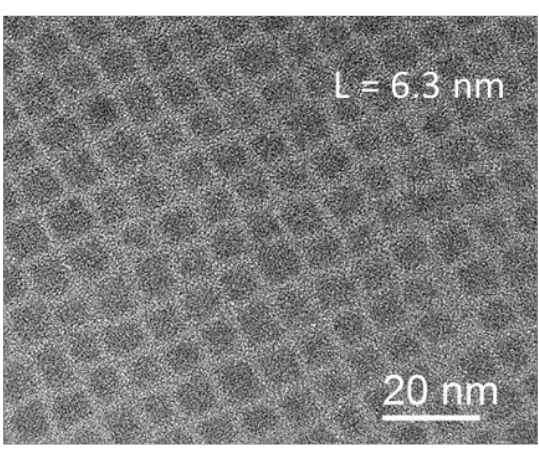

b

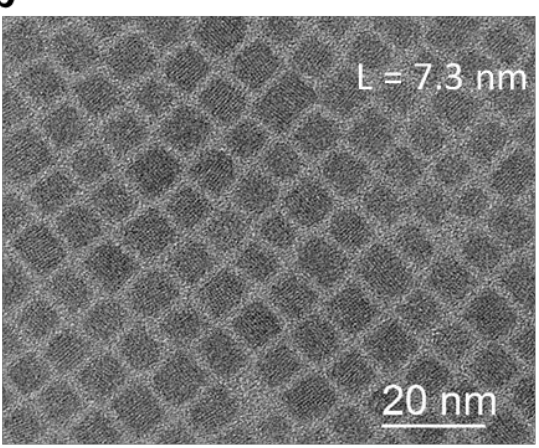

d

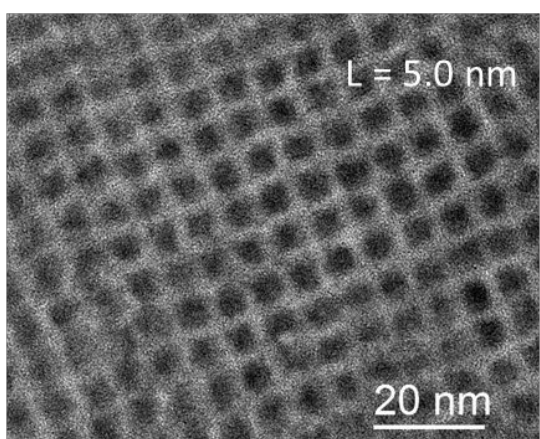

Figure S1. TEM images of $\mathrm{CsPbI}_{3}$ QDs of varying sizes. The average edge lengths of these QDs are indicated.
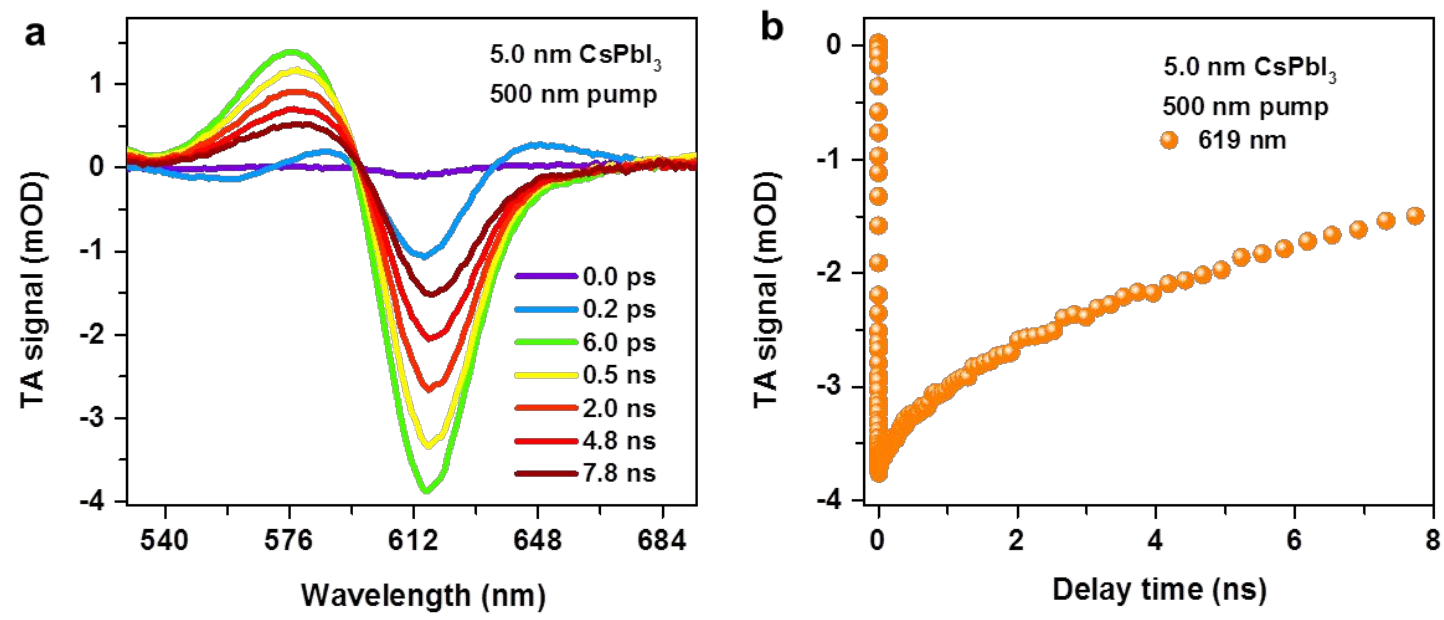

Figure S2. TA of $5.0 \mathrm{~nm} \mathrm{CsPbI} \mathbf{~}_{3}$ QDs. (a) TA spectra of $5.0 \mathrm{~nm} \mathrm{CsPbI}{ }_{3}$ QDs at indicated delays following the pump pulses of $500 \mathrm{~nm}$. (b) TA kinetics probed at the exciton bleach $(\sim 619 \mathrm{~nm})$. 

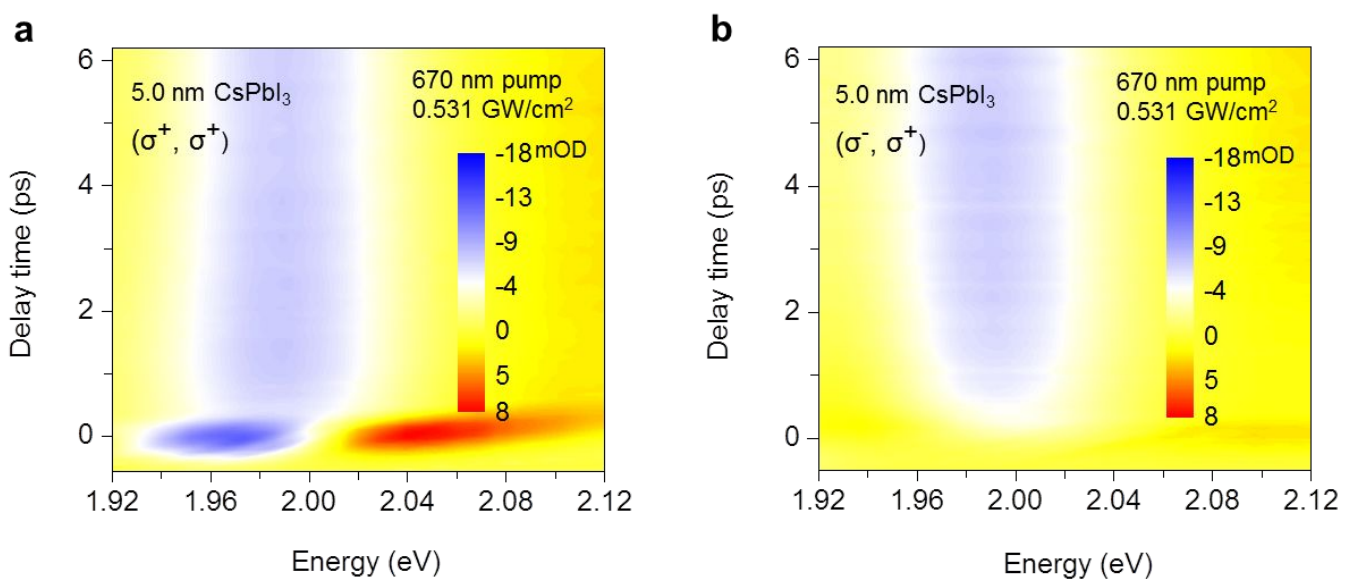

Figure S3. Spectral subtraction. 2D TA spectra of 5.0 nm CsPbI3 QDs dispersed in hexane measured with (a) co- $\left(\sigma^{+}, \sigma^{+}\right)$and (b) counter-circularly $\left(\sigma^{-}, \sigma^{+}\right)$polarized pump and probe pulses. The pump pulse is at $670 \mathrm{~nm}$ and the pump intensity is 0.531 $\mathrm{GW} / \mathrm{cm}^{2}$. The exciton bleach feature arising from two-photon absorption can be observed in both (a) and (b) and can be removed by taking a subtraction between (a) and (b).
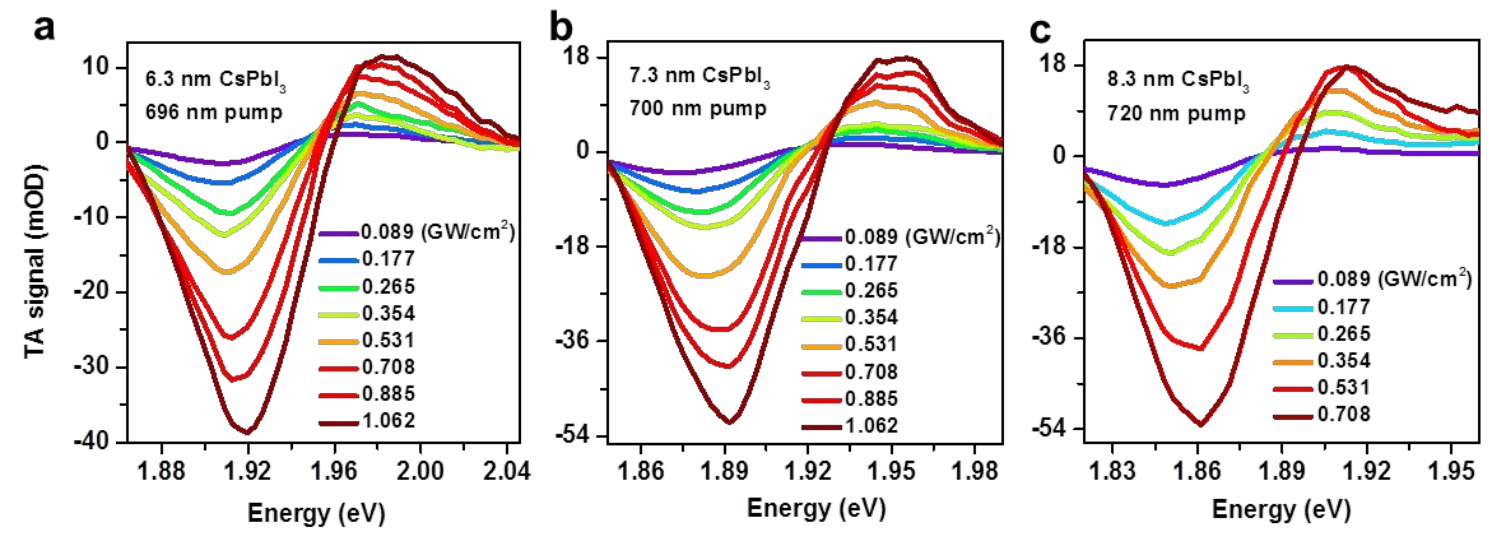

Figure S4. OSE of other size $\mathrm{CsPbI}_{3}$ QDs. Pump intensity dependent OSE spectra of (a) 6.3 , (b) 7.3 and (c) $8.3 \mathrm{~nm} \mathrm{CsPbI}{ }_{3}$ QDs at time zero (signal maxima). The pump wavelengths and intensities are indicated. 

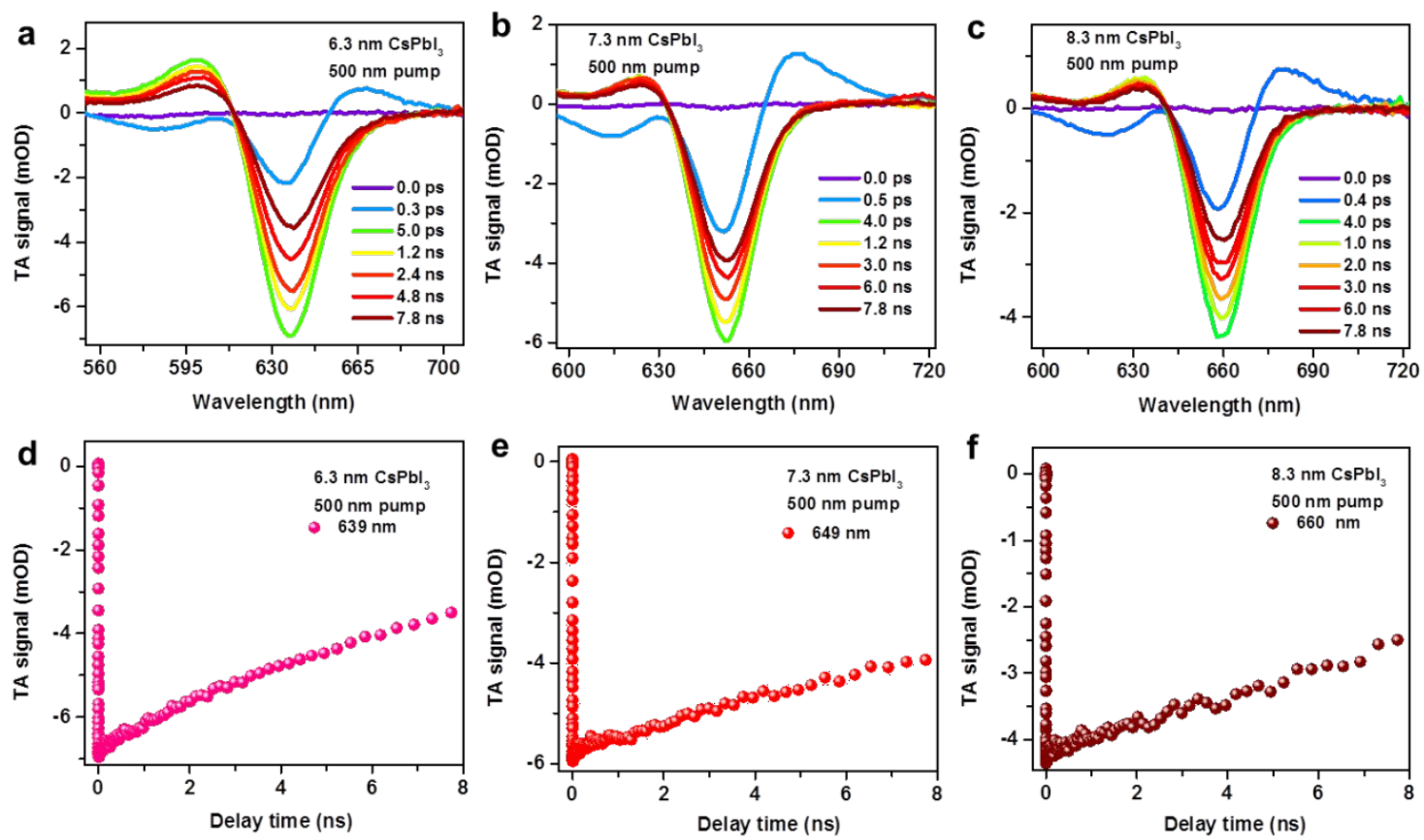

Figure S5. TA of other size $\mathbf{C s P b I}_{3}$ QDs. (a-c) TA spectra of (a) 6.3, (b) 7.3 and (c) $8.3 \mathrm{~nm} \mathrm{CsPbI}$ QDs at indicated delays following the pump pulses of $500 \mathrm{~nm}$. (d-f) TA kinetics probed at the exciton bleach of (a) 6.3, (b) 7.3 and (c) $8.3 \mathrm{~nm} \mathrm{CsPbI}$ QDs.

a

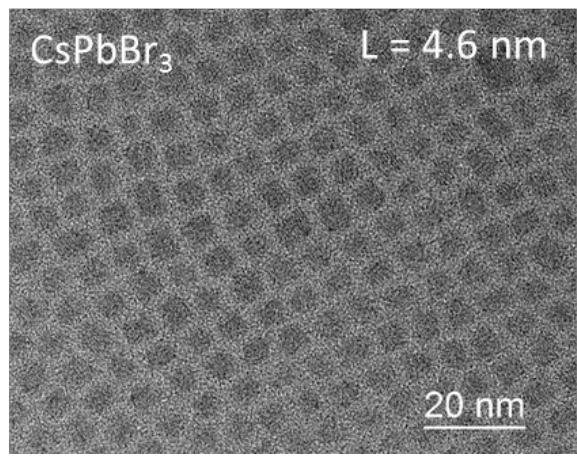

b

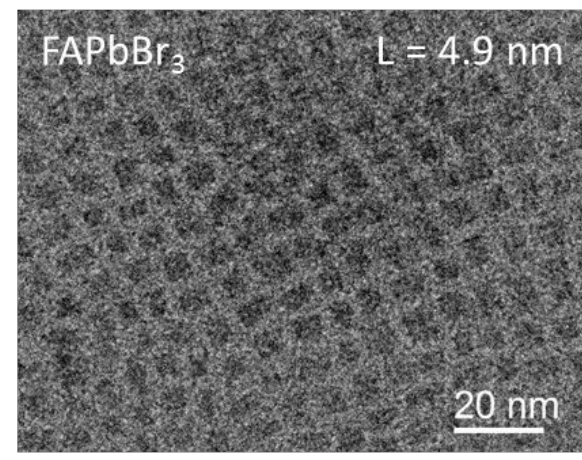

Figure S6. TEM images of (a) $\mathrm{CsPbBr}_{3}$ and (b) $\mathrm{FAPbBr}_{3}$ QDs. The average edge lengths of these QDs are indicated. 

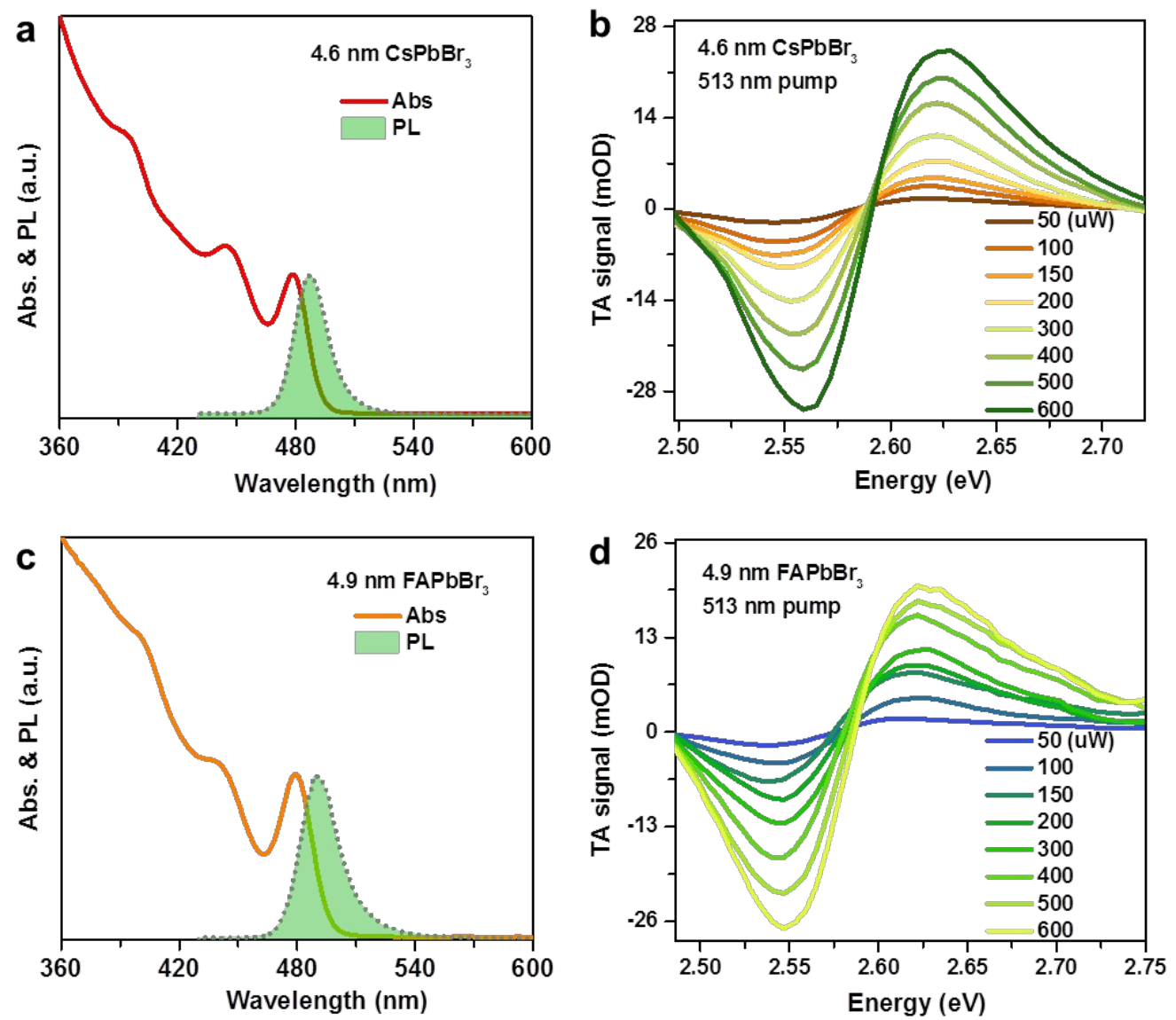

Figure S7. Absorption and OSE of $\mathrm{CsPbBr}_{3}$ and $\mathrm{FAPbBr}_{3}$ QDs. (a) Absorption (red solid lines) and photoluminescence (PL; green shadings) spectra of $4.6 \mathrm{~nm}$ $\mathrm{CsPbBr}_{3}$ QDs. (b) Pump intensity dependent OSE spectra of $4.6 \mathrm{~nm} \mathrm{CsPbBr}_{3}$ QDs at time zero (signal maxima). The pump wavelength and intensities are indicated. (c,d) The same plots as (a) and (b) but for $4.9 \mathrm{~nm} \mathrm{FAPbBr} 3$ QDs. 

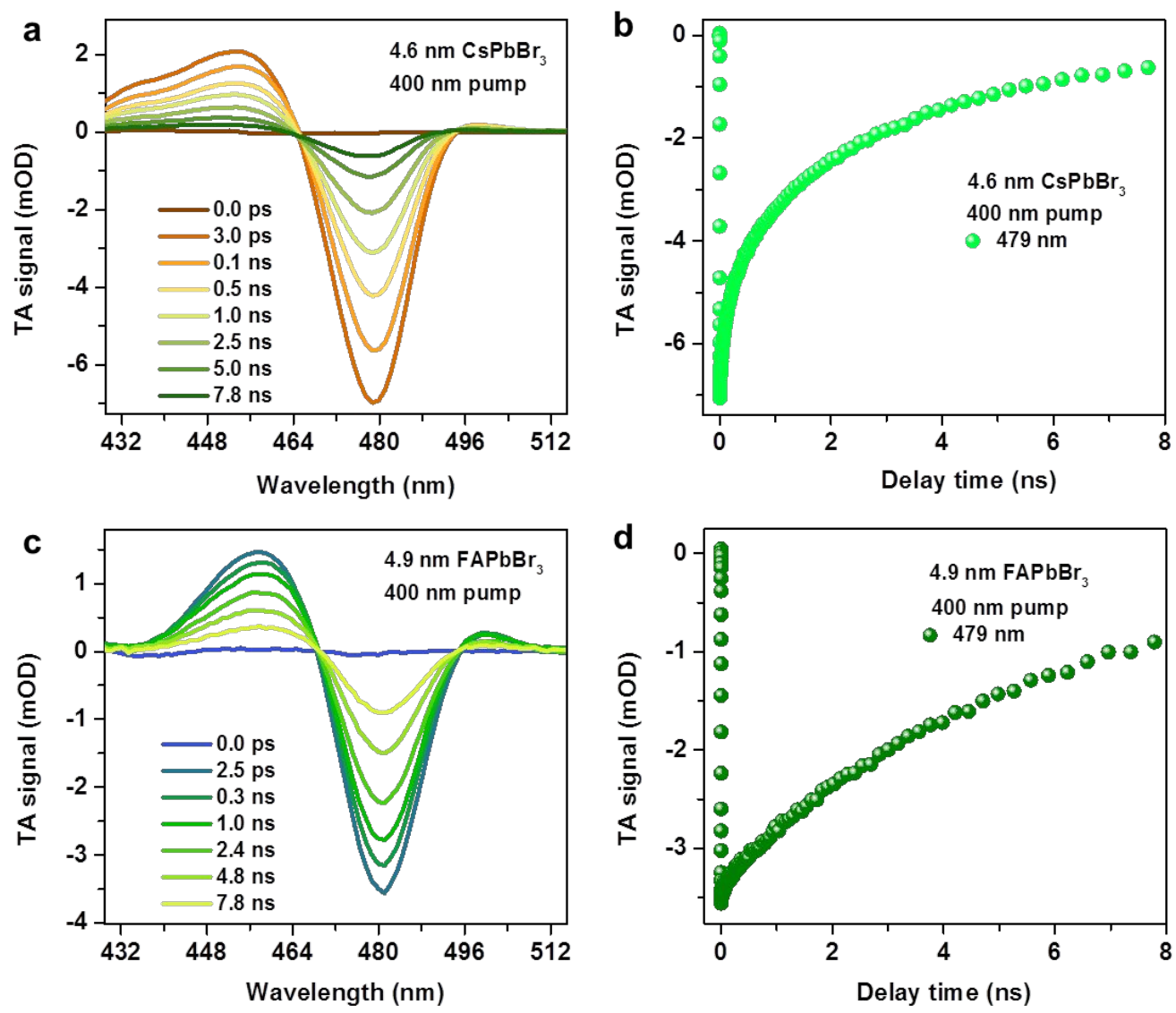

Figure S8. TA of $\mathrm{CsPbBr}_{3}$ and $\mathrm{FAPbBr}_{3}$ QDs. (a, b) TA spectra of (a) $4.6 \mathrm{~nm}$ $\mathrm{CsPbBr}_{3}$ QDs and (b) $4.9 \mathrm{~nm} \mathrm{FAPbBr}_{3}$ QDs. at indicated delays following the pump pulses of $400 \mathrm{~nm}$. (c, d) TA kinetics probed at the exciton bleach of (c) $4.6 \mathrm{~nm}$ $\mathrm{CsPbBr}_{3}$ QDs and (d) $4.9 \mathrm{~nm} \mathrm{FAPbBr} 3$ QDs. 


\section{Experimental Methods}

Synthesis of perovskite QDs We synthesized $\mathrm{CsPbI}_{3}$ QDs by modifying previously reported procedures..$^{1,2}$ The synthesis started with the preparation of Cs-oleate precursors. $0.25 \mathrm{~g} \mathrm{Cs}_{2} \mathrm{CO}_{3}, 0.98 \mathrm{~mL}$ oleic acid (OA), and $9 \mathrm{~mL}$ 1-octadecene (ODE) were loaded into a $50 \mathrm{~mL}$ 3-neck flask and vacuum-dried for $1 \mathrm{~h}$ at $120^{\circ} \mathrm{C}$ using a Schlenk line. The mixture was heated under argon atmosphere to $150{ }^{\circ} \mathrm{C}$ until all the $\mathrm{Cs}_{2} \mathrm{CO}_{3}$ was dissolved. The Cs-oleate precursor solution was kept at $100{ }^{\circ} \mathrm{C}$ to prevent precipitation of Cs-oleate out of ODE. In another $25 \mathrm{~mL}$ 3-neck flask, a precursor solution of $\mathrm{Pb}$ and $\mathrm{I}$ was prepared by dissolving $\mathrm{PbI}_{2}(120 \mathrm{mg})$ and $\mathrm{ZnI}_{2}$ (180-500 $\mathrm{mg})$ in a mixture of ODE $(5 \mathrm{~mL})$, oleyl amine (OAm; $2 \mathrm{~mL})$ and Bis (2,4,4-trimethylpentyl) phosphinic acid (TMPPA, $2 \mathrm{~mL}$ ). After the precursor solution of $\mathrm{Pb}$ and $\mathrm{I}$ was vacuum-dried for $1 \mathrm{~h}$ at $120^{\circ} \mathrm{C}$, it was set to the reaction temperature under argon atmosphere. The reaction temperature was varied depending on the desired QD sizes $\left(90{ }^{\circ} \mathrm{C}\right.$ for $5.0 \mathrm{~nm}, 110{ }^{\circ} \mathrm{C}$ for $6.3 \mathrm{~nm}, 120{ }^{\circ} \mathrm{C}$ for $7.3 \mathrm{~nm}, 140{ }^{\circ} \mathrm{C}$ for $8.3 \mathrm{~nm})$. The reaction was quenched by cooling the flask in an ice bath. When the crude solution was cooled down to room temperature, the product was centrifuged at $3500 \mathrm{rpm}$ for $15 \mathrm{~min}$ to remove the unreacted salts as the precipitate, and the QDs dispersed in the supernatant were collected. Methyl acetate was then used as the antisolvent for the isolation and size selectivity (when necessary) of $\mathrm{CsPbI}_{3}$ QDs. The precipitated QDs were used for further characterization after dispersing in hexane. 
$\mathrm{CsPbBr}_{3}$ and $\mathrm{FAPbBr}_{3}$ QDs were synthesized according to our recently reported methods. $^{3-6}$

Pump-probe experiment. The femtosecond pump-probe TA measurements were performed using a regenerative amplified Ti:sapphire laser system (Coherent; $800 \mathrm{~nm}$, $6 \mathrm{~mJ}$ per pulse, and $1 \mathrm{kHz}$ repetition rate) as the laser source and a Femto-100 spectrometer (TimeTech LLC) as the spectrometer. The fundamental beam is then split into two beams with a beam splitter. The transmitted part was used to pump a TOPAS OPA to generated the pump beam which was chopped at a rate of $500 \mathrm{~Hz}$. The reflected $800 \mathrm{~nm}$ beam was sent through a delay stage and attenuated with a neutral density filter and focused into a $2 \mathrm{~mm}$ thick sapphire or $\mathrm{CaF}_{2}$ window to generate a white light continuum (WLC) used as the probe beam. The pump and probe beams were focused and overlapped onto the sample. Circularly polarized pump and probe pulses were controlled separately by two sets of broadband polarizing beam splitter cubes (400-700 nm, Thorlabs) and quarter-wave plates $(350-850 \mathrm{~nm}$, Thorlabs). The pump beam size was $300 \mu \mathrm{m}$ and the instrument response time was $\sim 200$ fs. Samples were vigorouly stirred during the measurements to avoid photodamaging.

\section{Calculation of the pulse intensity}

The pulse intensity is the ratio of average pulse energy density to the pulse duration ( $\sim 200 \mathrm{fs}$ ) which is the full width at half maximum of TA instrument response function (IRF). First, we can estimate the pulse energy density $\left(\rho, \mu \mathrm{J} / \mathrm{cm}^{2}\right)$ according to the laser power $(\mu \mathrm{W})$, laser repetition rate $(1000 \mathrm{~Hz})$ and beam size (radius $\sim 0.03 \mathrm{~cm}$, 
area $A \sim 2.83 \times 10^{-3} \mathrm{~cm}^{2}$ ). The calculation details are as follows taking $50 \mu \mathrm{W}$ for example.

The pulse energy density :

$$
\begin{gathered}
E=\frac{50 \times 10^{-6}}{1000}=5.0 \times 10^{-8} \mathrm{~J} \\
\rho=\frac{E}{A}=\frac{5.0 \times 10^{-8}}{2.83 \times 10^{-7}}=0.177 \mathrm{~J} / \mathrm{m}^{2}=17.7 \mu \mathrm{J} / \mathrm{cm}^{2}
\end{gathered}
$$

The pulse intensity:

$$
I_{0}=\frac{\rho}{\tau}=\frac{0.177}{2.0 \times 10^{-13}}=8.85 \times 10^{11} \mathrm{~W} / \mathrm{m}^{2}=0.0885 \mathrm{GW} / \mathrm{cm}^{2}
$$

\section{Calcualtions of the spectral weight transfer (SWT) and OSE shift ( $\delta E)$}

For an absorption spectrum of an aribitury shape deseribed by a function $A(E)$, where $E$ is the energy and $E_{0}$ is the absorption peak, the transient change of the spectrum due to a small positive shift of $\delta E$ can be approximated by the first derivative of the function and $\delta E$ as following:

$$
\delta A(E)=A(E-\delta E)-A(E) \approx-\frac{d A(E)}{d E} \delta E
$$

Thus, for an analytical function $A(E), \delta E$ can be easily estimated using the first derivative of $A(E)$. For the case of an unknown function, however, $\delta E$ should be estimated through the so-called spectral weight transfer (SWT) approach:7,8

$$
\mathrm{SWT}=\int_{0}^{E_{0}} \delta A(E) d E
$$

Combining Eqs. 1 and 2, the SWT can be derived as:

$$
\mathrm{SWT}=-\delta E \int_{0}^{E_{0}} \frac{d A(E)}{d E} d E=-\delta E\left(A\left(E_{0}\right)-A(0)\right)
$$

Thus, the energy shift $\delta E$ due to the OSE can be calculated as:

$$
\delta E=-\frac{\mathrm{SWT}}{A\left(E_{0}\right)}
$$




\section{References for SI:}

(1) Dong, Y.; Qiao, T.; Kim, D.; Parobek, D.; Rossi, D.; Son, D. H. Precise Control of Quantum Confinement in Cesium Lead Halide Perovskite Quantum Dots via Thermodynamic Equilibrium. Nano Lett. 2018, 18, 3716-3722.

(2) Wang, C.; Chesman, A. S. R.; Jasieniak, J. J. Stabilizing the cubic perovskite phase of $\mathrm{CsPbI} 3$ nanocrystals by using an alkyl phosphinic acid. Chem. Commun. 2017, 53, 232-235.

(3) Li, Y.; Ding, T.; Luo, X.; Chen, Z.; Liu, X.; Lu, X.; Wu, K. Biexciton Auger recombination in mono-dispersed, quantum-confined $\mathrm{CsPbBr} 3$ perovskite nanocrystals obeys universal volume-scaling. Nano Res. 2019, 12, 619-623.

(4) Li, Y.; Lai, R.; Luo, X.; Liu, X.; Ding, T.; Lu, X.; Wu, K. On the absence of a phonon bottleneck in strongly-confined $\mathrm{CsPbBr} 3$ perovskite nanocrystals. Chem. Sci. 2019, 10, 5983-5989.

(5) Luo, X.; Lai, R.; Li, Y.; Han, Y.; Liang, G.; Liu, X.; Ding, T.; Wang, J.; Wu, K. Triplet Energy Transfer from $\mathrm{CsPbBr} 3$ Nanocrystals Enabled by Quantum Confinement. J. Am. Chem. Soc. 2019, 141, 4186-4190.

(6) Li, Y.; Ding, T.; Luo, X.; Tian, Y.; Lu, X.; Wu, K. Synthesis and Spectroscopy of Monodispersed, Quantum-Confined FAPbBr3 Perovskite Nanocrystals. Chem. Mat. 2020, 32, 549-556.

(7) Giovanni, D.; Chong, W. K.; Dewi, H. A.; Thirumal, K.; Neogi, I.; Ramesh, R.; Mhaisalkar, S.; Mathews, N.; Sum, T. C. Tunable room-temperature spin-selective optical Stark effect in solution-processed layered halide perovskites. Sci. Adv. 2016, 2, e1600477.

(8) Sie, E. J.; McIver, J. W.; Lee, Y.-H.; Fu, L.; Kong, J.; Gedik, N. Valley-selective optical Stark effect in monolayer WS2. Nat. Mater. 2014, 14, 290. 